\title{
Agrin, a novel basement membrane component in human and rat liver, accumulates in cirrhosis and hepatocellular carcinoma
}

\author{
Péter Tátrai ${ }^{1}$, József Dudás ${ }^{2}$, Enkhjargal Batmunkh ${ }^{3}$, Miklós Máthé ${ }^{1}$, Attila Zalatnai ${ }^{1}$, \\ Zsuzsa Schaff ${ }^{3}$, Giuliano Ramadori ${ }^{2}$ and Ilona Kovalszky ${ }^{1}$ \\ ${ }^{1} 1$ st Institute of Pathology and Experimental Cancer Research, Semmelweis University, Budapest, Hungary; \\ ${ }^{2}$ Department of Gastroenterology and Endocrinology, Georg August University of Göttingen, Göttingen, \\ Germany and ${ }^{3}$ 2nd Institute of Pathology, Semmelweis University, Budapest, Hungary
}

\begin{abstract}
Agrin is a multifunctional heparan sulfate proteoglycan originally discovered in the neuromuscular junctions and later observed in numerous other localizations. The presence of agrin in the liver, either healthy or diseased, has formerly not been reported. We detected agrin in minor amounts in the basement membranes of blood vessels and bile ducts in the healthy liver. The proliferation of bile ductules and the formation of new septal blood vessels in liver cirrhosis, as well as neoangiogenesis in the hepatocellular carcinoma (HCC) result in a dramatic increase in the quantity of agrin. Vascular and peribiliary basement membranes were strongly immunopositive for agrin in 29/29 human liver specimens with cirrhosis and HCC. However, sinusoidal walls of regenerative nodules in the cirrhotic liver consistently remained negative. Given the selectivity of agrin for tumor microvessels, agrin immunohistochemistry may prove helpful in recognizing malignant transformation in cirrhotic livers. Similar immunohistochemical observations were made on the liver of rats exposed to a combined cirrhosis/HCC induction treatment. In both human and rats, agrin probably originates from activated myofibroblasts, vascular smooth muscle cells and biliary epithelial cells. Increased agrin expression in human specimens, in the liver of $4 / 4$ treated rats, as well as in isolated rat liver mesenchymal cells was verified by quantitative RT-PCR. Considering that agrin binds various growth factors, and it directly interacts with cell membrane receptors such as $\alpha_{v}$-integrins, we hypothesize a stimulatory role for agrin in neoangiogenic processes such as tumor vascularization, and a supportive role in bile ductule proliferation.
\end{abstract}

Laboratory Investigation (2006) 86, 1149-1160. doi:10.1038/labinvest.3700475; published online 18 September 2006

Keywords: agrin; basement membrane; cirrhosis; heparan sulfate proteoglycan; hepatocellular carcinoma; neoangiogenesis

In chronic liver diseases, several proteoglycans (PGs) have been described to accumulate in the matrix of the expanding scar tissue, in the basement membranes of proliferated bile ducts and capillarized sinusoids, as well as in the walls of newly formed blood vessels. Elevated amounts of decorin and perlecan have been observed in the extracellular matrix of acutely and chronically damaged rat liver. ${ }^{1,2}$ Similarly, decorin is an early marker of fibrogenesis in human livers with chronic hepatitis. ${ }^{3}$

Correspondence: P Tátrai, MSc, 1st Institute of Pathology and Experimental Cancer Research, Semmelweis University, Üllöi út 26, H-1085, Budapest, Hungary.

E-mail: tatpeter@korb1.sote.hu

Received 22 March 2006; revised and accepted 10 August 2006; published online 18 September 2006
Decorin and biglycan, both normally limited to the space of Disse, peribiliary matrix and vessel walls, are extensively deposited in fibrotic areas, while PG-100, the PG form of colony-stimulating factor 1 appears in a novel biliary localization. ${ }^{4}$

The tumor-specific matrix of hepatocellular carcinoma (HCC), mainly secreted by stromal myofibroblast-like cells, microvascular endothelial cells and by the tumor cells themselves, is characterized by a PG milieu that greatly differs from that of both the normal and the injured but nontumorous liver tissue. The matrix PGs perlecan and decorin have been found not only in the fibrotic liver, but in tumors, too. ${ }^{5,6}$ Perlecan promotes tumor growth and angiogenesis $;^{7,8}$ decorin, in contrast, has antitumor effects: stromal decorin expression is a part of the host defense reaction against the tumor. ${ }^{9}$ Thus, 
altered PG composition of the extracellular matrix is characteristic of chronic liver diseases, and PGs are key players in a multitude of pathological processes.

In the present study, we report a marked increase in the quantity of agrin, a multidomain heparan sulfate proteoglycan (HSPG), in the diseased liver. Most of our current knowledge on the structure and functions of agrin was summarized in 2003 by Gabriela Bezakova and Markus Ruegg ${ }^{10}$, pioneers of agrin research. With relevance to our subject, the long N-terminal splice isoform of agrin is secreted into basement membranes, where, by binding to laminins with its N-terminal end and simultaneously to cell surface receptors such as $\beta$-dystroglycan or integrins ${ }^{10-14}$ with its C-terminus, it mechanically anchors the cortical actin network of the adjacent cell to the laminin network of the basement membrane. Furthermore, agrin is able to activate specific intracellular signaling pathways, in part directly (via kinase receptors like musclespecific kinase), in part indirectly (eg via integrins). ${ }^{10,11}$

Agrin, either secreted or membrane-bound, is known to play multiple roles in both neural and non-neural tissues. At neuromuscular junctions ${ }^{15}$ and in the brain, ${ }^{16}$ it is indispensable for synaptic organization; agrin is also a key factor in axonal growth, and in the formation and maintenance of the blood-brain barrier. ${ }^{17,18}$ Agrin is also needed for the aggregation of lipid rafts in the membrane of T cells, hence, for 'immunological synapse' forming. ${ }^{19}$ In the renal cortex, agrin is critical for podocyte attachment to the glomerular basement membrane. ${ }^{11,20} \mathrm{~A}$ possible role for agrin in the liver has previously not been proposed, probably because it was believed to be absent from this organ. ${ }^{12}$ After the identification of agrin in PG extracts from cirrhotic livers and HCCs, we first intended to describe its distribution in the tissue and its putative cellular sources, as well as to identify some of its actual or potential molecular interactions. For these purposes, we performed immunohistochemical and immunofluorescence studies on human tissue specimens and on cultured human and rat cells. Secondly, myofibroblasts and hepatic stellate cells (HSC) from rat liver were examined as candidates for agrin production, modeling activated mesenchymal cells that play a prominent role in fibrogenesis ${ }^{21}$ and tumoral stromal reaction. ${ }^{22}$ Moreover, agrin expression on the mRNA and protein level was also determined in a rat cirrhosis/hepatocarcinogenesis model to investigate whether a rodent system may be suitable for future functional research on the role of agrin in liver diseases.

\section{Materials and methods}

\section{Patient Materials}

For the purposes of immunohistochemistry and PG isolation, 29 surgical specimens from patients undergone partial hepatectomy for HCC and four healthy livers were obtained from surgical, transplantation and forensic departments of the Semmelweis University, Budapest, Hungary, and the Department of Cytopathology, Deutsches Krebsforschungszentrum, Heidelberg, Germany. HCC was associated with cirrhosis in all patients. Regarding the etiologies of liver disease, alcohol abuse was reported in seven cases, while hepatitis B-virus infection was diagnosed in 12 cases, hepatitis C-virus infection in seven cases, and hepatitis $\mathrm{B} / \mathrm{C}$ co-infection in one case; in two patients, the disease was cryptogenic. Other fresh surgical specimens at the Department of Gastroenterology and Endocrinology, University Clinic, Göttingen, Germany were snap-frozen and later used for RT-PCR. All experiments involving material of human origin were performed with the permission of the regional ethical committee and with the written consent of the patients whose tissues were submitted to special studies after establishing the diagnosis.

\section{Animal Experimentation}

Wistar rats were provided by Charles River (Sulzfeld, Germany). Livers from untreated animals were used for the isolation of rat liver myofibroblasts (rMFs) and HSCs (see Cell Culture). Four male rats were subjected to DPC (diethyl-nitrosamine/phenobarbital/carbon tetrachloride) treatment. This simultaneous cirrhosis/HCC induction protocol involves a single administration of diethyl-nitrosamine in a necrogenic dose, followed by a 6-week combined treatment with phenobarbital and carbon tetrachloride, as described by Zalatnai and Lapis. ${ }^{23}$ All animals when killed at week 16 had histologically proven cirrhosis and multifocal HCC. Animals received humane care in compliance with the institution's guidelines and National Institutes of Health guidelines.

\section{Cell Culture}

rMF and HSC were isolated and the purity of the preparations was assessed as described elsewhere. ${ }^{21,24,25}$ Human dermal microvasculature endothelial cells (HMVEC-d) were purchased from Cambrex (Walkersville, MD, USA), and cultured in the supplemented medium recommended by the company. rMF at passage 4 (mRNA quantitation) and 13-14 (immunofluorescence, supernatant conditioning), HSC at day 7 after isolation, and HMVEC$\mathrm{d}$ at passage 5 were used in the experiments.

\section{PG Isolation}

PGs were isolated from three human liver samples (nontumorous cirrhotic tissue, HCC and normal 
Table 1 Primary antibodies, applications and corresponding dilutions

\begin{tabular}{|c|c|c|}
\hline Antibody description & Application/dilution & Manufacturer \\
\hline Anti-bovine/human agrin, clone 7E12 & $\begin{array}{l}\text { WB/1:500 } \\
\mathrm{IHC} / 1: 100 \\
\mathrm{iIF} / 1: 50\end{array}$ & Chemicon $^{\mathrm{a}}$ \\
\hline Anti-rat agrin, goat polyclonal & $\mathrm{iIF} / 2 \mu \mathrm{g} / \mathrm{ml}$ & R\&D Systems ${ }^{b}$ \\
\hline Anti-rat agrin, goat polyclonal & $\mathrm{WB} / 1: 250$ & Santa Cruz ${ }^{\mathrm{C}}$ \\
\hline Anti-human cytokeratin, FITC conjugate, clone MNF116 & $\mathrm{dIF} / 1: 10$ & Dako $^{\mathrm{d}}$ \\
\hline $\begin{array}{l}\text { Anti-human fibroblast growth factor, basic, goat } \\
\text { polyclonal }\end{array}$ & $\mathrm{iIF} / 1: 50$ & R\&D Systems \\
\hline Anti-human integrin, $\alpha_{v}$, clone P2W7 & $\mathrm{iIF} / 10 \mu \mathrm{g} / \mathrm{ml}$ & R\&D Systems \\
\hline Anti-human laminin, rabbit polyclonal & $\mathrm{iIF} / 1: 200$ & Dako \\
\hline Anti-human perlecan, clone 7B5 & IHC/1:10 & Zymed $^{\mathrm{e}}$ \\
\hline Anti-rat RECA-1, clone HIS52 & $\mathrm{iIF} / 1: 50$ & Serotec $^{\mathrm{f}}$ \\
\hline Anti-human smooth muscle $\alpha$-actin, clone $1 \mathrm{~A} 4$ & $\mathrm{iIF} / 1: 100$ & Dako \\
\hline
\end{tabular}

WB, Western blot; IHC, immunohistochemistry; iIF, indirect immunofluorescence; dIF, direct immunofluorescence.

${ }^{\mathrm{a} C h e m i c o n}$ International Inc., Temecula, CA, USA.

${ }^{\mathrm{b}} \mathrm{R} \& \mathrm{D}$ Systems, Abingdon, UK.

${ }^{\mathrm{c}}$ Santa Cruz Biotechnology, Santa Cruz, CA, USA.

${ }^{\mathrm{d}}$ Dako, Glostrup, Denmark.

${ }^{\mathrm{e}}$ Zymed, South San Francisco, CA, USA.

${ }^{\mathrm{f}}$ Serotec, Oxford, UK.

liver tissue), and from bovine kidney cortex, according to the slightly modified method of Lyon and Gallagher. ${ }^{26}$ Briefly, proteins from tissue homogenates were extracted with $4 \mathrm{~mol} / \mathrm{l}$ guanidine hydrochloride. Subsequently, all proteins except polyanionic PGs were precipitated by adding trichloroacetic acid up to $10 \% \mathrm{w} / \mathrm{v}$. After centrifugation, the supernatant was pH-neutralized and dialyzed with several changes against a concentrated $(7 \mathrm{~mol} / \mathrm{l})$, buffered urea solution containing protease inhibitors. (In the case of bovine kidney cortex, trichloroacetic acid precipitation was omitted.) Dialyzed samples were applied to DEAE52 Servacel (Serva, Heidelberg, Germany) columns, which were then washed with a two-step salt gradient $(0.1 \mathrm{~mol} / \mathrm{l}, 0.2 \mathrm{~mol} / \mathrm{l}$ sodium chloride) in urea buffer. PGs were eluted with urea buffer containing $0.8 \mathrm{~mol} / \mathrm{l}$ sodium chloride, and precipitated with cold ethanol before use. Alternatively, for magnetic bead isolation, PGs were directly dialyzed into PBS containing protease inhibitors.

\section{Antibodies}

Three anti-agrin antibodies, one monoclonal and two polyclonals, were used in various applications. The monoclonal antibody clone 7E12, now available as MAB 458 (Chemicon International Inc., Temecula, CA, USA), was raised against an HSPG isolated from bovine renal glomerular basement membrane by Kemeny et al. ${ }^{27}$ The specificity of this antibody for agrin was verified by our group as follows. MAB 458 was covalently crosslinked to anti-mouse IgGcoated Dynabeads (Invitrogen, Carlsbad, CA, USA) and the beads were incubated with PGs isolated from bovine kidney cortex. Subsequently, the beads were heat-denatured in sample buffer, and the supernatant was run on a $5-15 \%$ gradient PA gel. A single high molecular weight, Alcian blue-stained smear was excised and sent for mass spectrometry analysis. Similar to agrin predicted protein of Bos taurus, showing a high degree of homology with human agrin, was identified in the sample on the basis of three peptides (IFFVNPAPPYLWPAHK, FGALCEAETGR, CEPGFWNFR).

Anti-agrin (R-20) goat polyclonal antibody from Santa Cruz Biotechnology (Santa Cruz, CA, USA) was raised against an epitope of rat agrin mapping near the C-terminus. Anti-agrin goat PAb from R\&D Systems (Abingdon, UK) was raised against rat recombinant agrin produced in insect cell culture. In our experiments, the latter antibody also reacted with human tissues. All antibodies, their applications and the corresponding dilutions or concentrations are listed in Table 1.

\section{Western Blot}

For liver PG Western blotting, PGs were isolated as described, and aliquots corresponding to $100 \mathrm{mg}$ of wet tissue were applied to a $5-15 \%$ gradient sodium dodecyl sulfate polyacrylamide gel. Proteins from rMF and HSC conditioned media were precipitated with cold ethanol, and aliquots containing $15 \mu \mathrm{g}$ of protein were applied per lane. Gels were blotted on PVDF membrane (Millipore, Bedford, MA, USA) overnight at $75 \mathrm{~mA}, 4^{\circ} \mathrm{C}$ in a wet blotter (Bio-Rad, Hercules, CA, USA). Incubations with primary antibodies plus $3 \% \mathrm{w} / \mathrm{v}$ bovine serum albumin (BSA), with biotinylated secondary antibodies (DakoCytomation, Glostrup, Denmark) plus $3 \% \mathrm{w} / \mathrm{v} \mathrm{BSA}$, and with peroxidase conjugated 
streptavidin-biotin complex (StreptABComplex/ HRP, DakoCytomation) were followed by chemiluminescent (SuperSignal West Pico, Pierce, Rockford, IL, USA) or chromogenic (3,3'-diaminobenzidine, Aldrich, Milwaukee, WI, USA) development.

\section{Immunohistochemistry and Immunofluorescence}

Immunoreactions were performed on tissue sections of snap-frozen human and rat livers, on sections of formalin-fixed, paraffin-embedded human liver surgical specimens prepared for histology, and on cultured cells. Frozen tissue sections and cells grown on glass coverslips were fixed in $-20^{\circ} \mathrm{C}$ methanol and $-20^{\circ} \mathrm{C}$ acetone. Paraffin sections were subjected to microwave antigen retrieval and enzymatic digestion with $0.01 \mathrm{mmol} / \mathrm{l}$ trypsin (Sigma, Steinheim, Germany). On frozen sections and cultured cells, nonspecific protein binding was blocked with $5 \% \mathrm{w} / \mathrm{v}$ BSA $+5 \% \mathrm{w} / \mathrm{v}$ nonspecific serum, and samples were incubated with the primary antibodies overnight at $4^{\circ} \mathrm{C}$. Immunoreactions were visualized either using secondary Alexa Fluor ${ }^{\circledR}$ antibodies (Molecular Probes, Eugene, OR, USA), followed by counterstaining with $4^{\prime}$,6-diamidino-2-phenylindole (Boehringer Mannheim, Mannheim, Germany), or using biotinylated secondary antibodies, ABC amplification and $\mathrm{DAB}$ Peroxidase Substrate Kit (Vector Laboratories, Burlingame, CA, USA), followed by hematoxylin counterstaining. For immunoperoxidase detection on liver histological sections, endogenous peroxidase was quenched first with $3 \% \mathrm{v} / \mathrm{v} \mathrm{H}_{2} \mathrm{O}_{2}\left(15 \mathrm{~min}, 25^{\circ} \mathrm{C}\right)$. Blocking and incubation with the primary antibody were performed as above. Following incubation with the appropriate biotinylated secondary antibodies (DakoCytomation), signal was amplified in three subsequent steps: (1) StreptABComplex/HRP (DakoCytomation); (2) biotinylated tyramide, diluted in $0.033 \% \mathrm{v} / \mathrm{v} \mathrm{H}_{2} \mathrm{O}_{2}$; (3) repeated ABC. As a chromogenic substrate, DAB (Aldrich) was used.

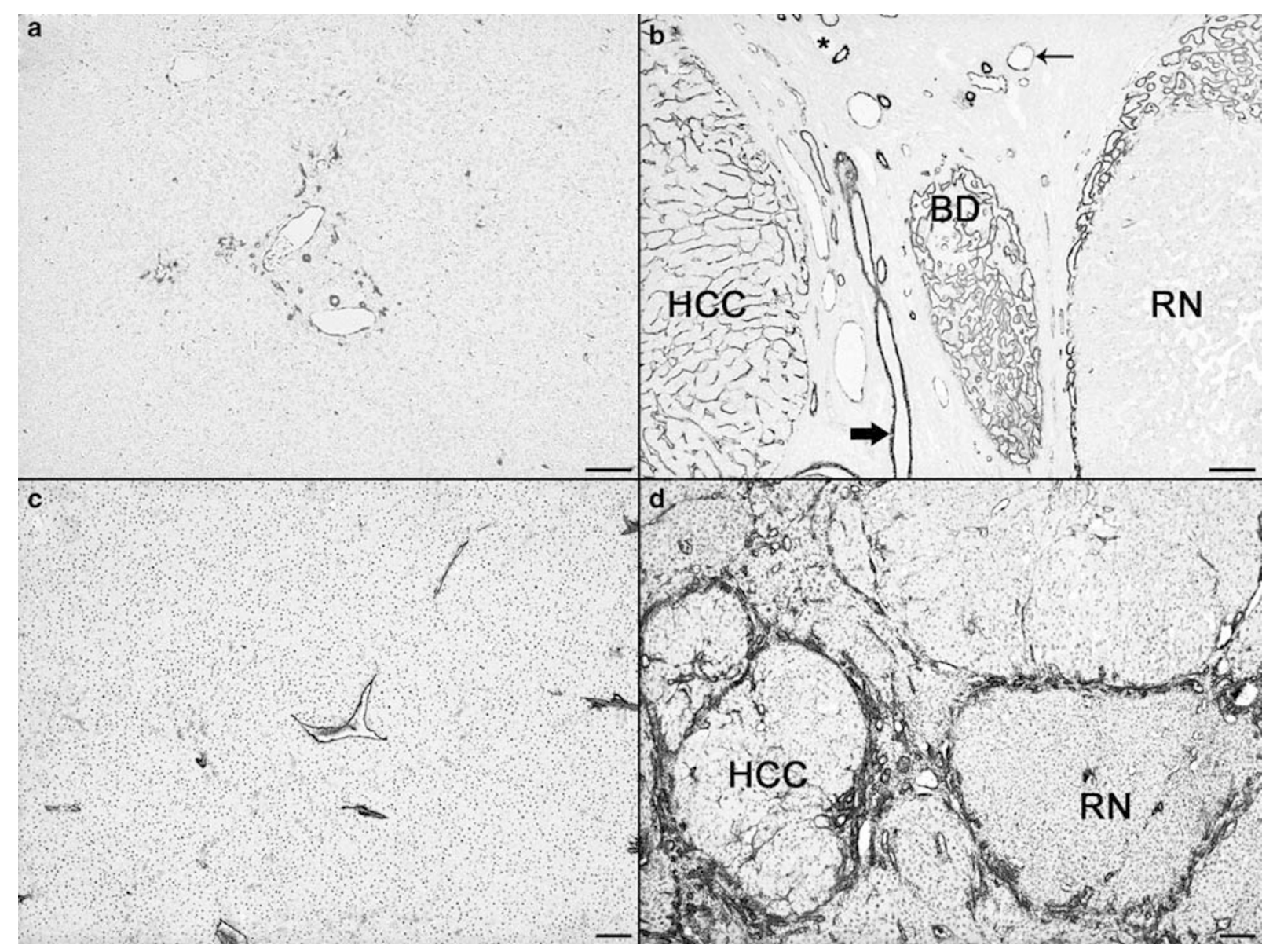

Figure 1 Agrin accumulates in the cirrhotic liver and in the HCC. (a, b) Immunohistochemistry for human agrin using the MAb clone 7E12; (c, d) immunohistochemistry for rat agrin using the PAb from R\&D. A small amount of agrin is detectable in the normal human (a) and rat (c) liver in the basement membranes of portal structures. (b) A cirrhotic human liver with large nodules of HCC. Intense agrin immunostaining is seen in the tumor (HCC), in the areas of proliferated bile ductuli (BD), and in the walls of arteries (thick arrow) and veins (thin arrow). However, no agrin appears in the regenerative nodules (RN). The walls of lymph vessels $\left({ }^{*}\right)$ contain no agrin. (d) The liver of a DPC-treated rat. Analogously, the basement membranes of bile ductuli and blood vessels within the fibrous septa show an intense agrin immunostaining. The neovascular walls inside the HCC nodules are moderately stained, while no immunoreaction appears in the regenerative nodules. Scale bars, $100 \mu \mathrm{m}$. 
Light micrographs were taken using a Nikon microscope equipped with an Olympus color CCD camera. Immunofluorescent preparations were photographed either with a Nikon microscope and a VDS Vosskühler (Osnabrück, Germany) monochromatic camera, or with a Nikon microscope equipped with a BioRad (Hercules, CA, USA) Laser Scanning Confocal System.

\section{Reverse Transcription-Polymerase Chain Reaction}

Total RNA was prepared from human surgical specimens, DPC-treated rat livers, isolated rMFs and HSCs using TRIzol Reagent (Invitrogen). Reverse transcription of RNA samples was performed with Superscript kit (Invitrogen), according to the instructions of the manufacturer. Real-time RT-PCR analysis of reverse transcripts was performed with the ABI Prism Sequence Detection System 7000 (Applied Biosystems, Foster City, CA, USA) following the manufacturer's instructions, using TaqMan Reaction Master Mix (Applied Biosystems) or Sybr Green Reaction Master Mix (Invitrogen), TaqMan Gene Expression Assays no. Rn00598349 (Applied Biosystems) for rat agrin, no. Hs00394748 for human agrin, no. Hs99999901 for human ribosomal RNA, as well as the following primers (MWG Biotech, Ebersberg, Germany; Applied Biosystems):

Rat agrin forward: $5^{\prime}$-GTGCCGTAAGGGATGAC TGT-3'

Rat agrin reverse: $5^{\prime}$-TAGCAGGACAGTTGGA GCCT-3'

Rat fibulin-2 forward: 5'-AGTCTGCCCAGGTAT CTCCCAA-3'

Rat fibulin-2 reverse: 5'-TCGCCAATAACGCGAC ACA-3'

Rat ubiquitin C forward: 5'-CACCAAGAAGGTCA AACAGGAA- $3^{\prime}$

Rat ubiquitin $\mathrm{C}$ reverse: $5^{\prime}$-AAGACACCTCCCCAT CAAACC- $3^{\prime}$.

\section{Results}

\section{The Accumulation of Agrin in the Diseased Liver}

Liver samples from four healthy human subjects and 29 patients with cirrhosis and HCC, as well as from two control and four DPC-treated rats were subjected to agrin immunohistochemistry. Agrin was detected in the normal human and rat liver, but only in small quantities in the basement membranes of portal structures, and never in the wall of the sinusoids (Figure 1a and c). Markedly elevated amounts of agrin were found in the cirrhotic liver tissue and in the HCC in both species (Figure 1b and d). This result was verified by immunoblotting using human liver PG extracts (Figure 2a), and by quantitative real-time RT-PCR experiments. In human pathological samples, agrin expression was a

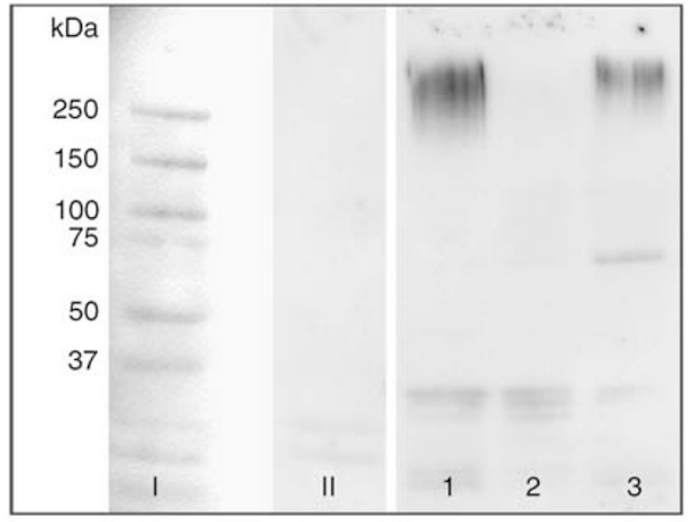

b
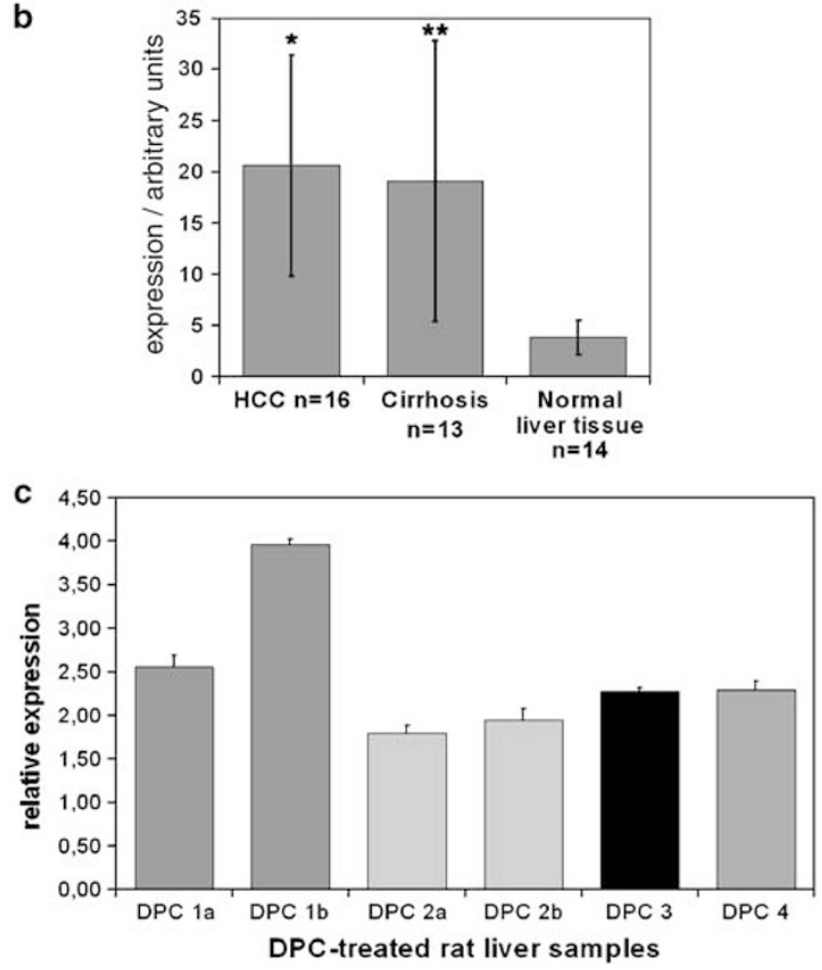

Figure 2 Verification of the immunohistochemical results by RT-PCR and Western blot. (a) Immunoblotting for human agrin using the MAb clone 7E12, chemiluminescent development. PG extracts of a HCC (lane 1) and a cirrhotic liver (lane 3) are positive for agrin, while this PG is not detectable in the extract of the normal liver tissue (lane 2). Specific immunoreaction appears as a smear above $250 \mathrm{kDa}$. Lane I, molecular weight ladder photographed under white light; lane II, MW ladder photographed in luminescence. (b) Quantitative RT-PCR on total RNA isolated from human liver surgical specimens. Samples removed from noncirrhotic livers with tumors, when distant from the tumor, and normal in histological appearance, were also regarded as normal. Agrin expression is significantly higher in both HCC and cirrhosis when compared to normal liver tissue $\left({ }^{*}\right.$ and ${ }^{* *}$ : Student's $t$-test, $P<0.001$ both), whereas the difference between cirrhotic tissue and HCC is not significant. Human ribosomal RNA was used as endogenous control. For the sake of convenience, expression values are given as $10^{6} \times 2^{-\Delta C_{\mathrm{t}}}$ where $\Delta C_{\mathrm{t}}$ means $C_{\mathrm{t}}$ (agrin) $-C_{\mathrm{t}}(\mathrm{rRNA})$. (c) Quantitative RT-PCR on total RNA isolated from the livers of one control and four DPC-treated rats. DPC1a and 1b, as well as DPC2a and 2b are parallel specimens taken from the same liver in order to assess intraorgan sampling error. Expression levels are compared to the normal sample, and calibrated by the household gene ubiquitin C. Treated livers exhibit a 1.8- to 4.0-fold increase in the expression of agrin. 

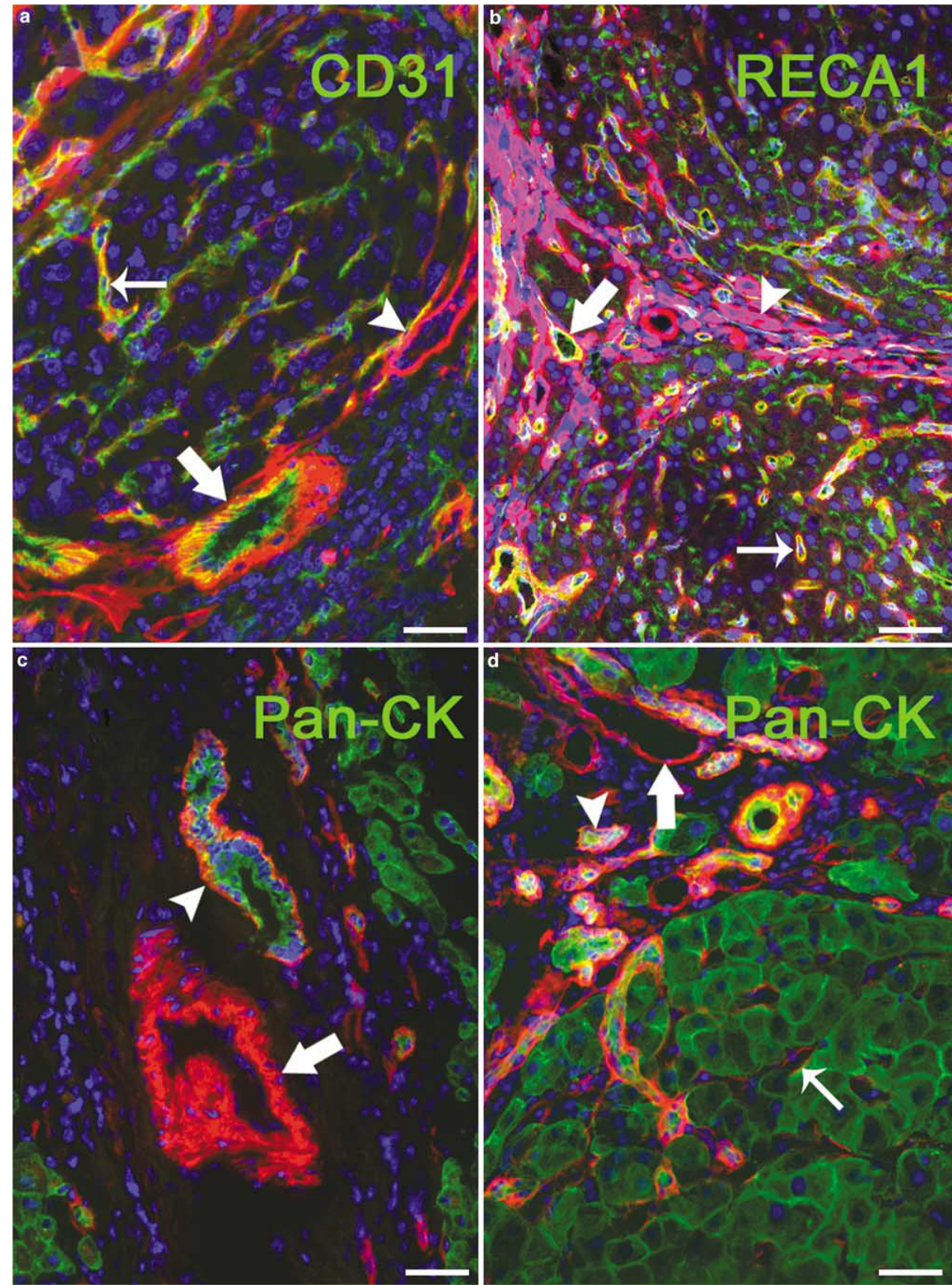


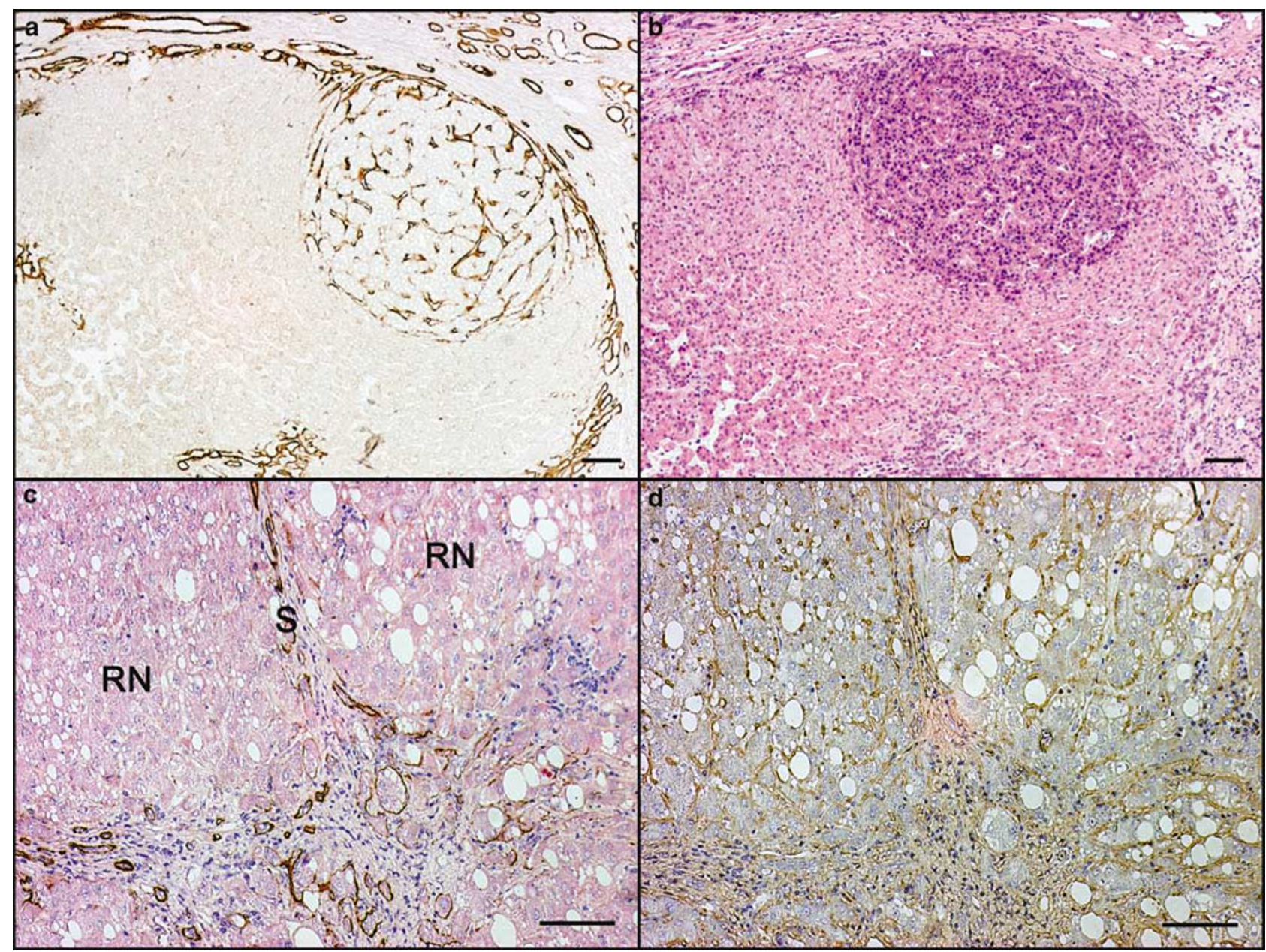

Figure 4 Agrin immunohistochemistry facilitates discrimination of neoplastic and regenerative nodules. (a) By immunohistochemistry using the monoclonal antibody clone 7E12, agrin appears in a 'nodule-in-nodule' type neoplastic focus in a human cirrhotic liver with HCC. (b) Hematoxylin-eosin stain of the area shown in (a). (c) In the cirrhotic liver, agrin is only deposited in the basement membranes of bile ductules and blood vessels within the fibrous septa (S) and never in the sinusoidal walls of regenerative nodules (RN). (d) Perlecan, in contrast, can occasionally be seen in the latter localization. Hence, agrin might be an even more reliable marker of tumor angiogenesis. Scale bars, $100 \mu \mathrm{m}$.

found to be significantly elevated in both cirrhosis and HCC when compared to the normal liver tissue (Student's $t$-test, $P<0.001$ both), whereas the difference between cirrhosis and HCC was not significant (Figure 2b). Increased expression of agrin, relative to the untreated control, was measured in the livers of DPC-treated rats as well (Figure 2c).

In both human and rats, agrin was seen in the basement membranes of bile ducts and blood vessel walls. The walls of lymphatic vessels showed no agrin immunoreaction. Vascular and biliary localizations were further verified by double immunofluorescent technique (Figure 3). In the HCC, agrin was deposited in basement membranes of the tumor microvasculature. On the other hand, agrin was absent from the wall of the sinusoids of the regenerative nodules, similarly to normal sinusoidal walls. Consequently, agrin can be considered as a marker of tumor angiogenesis. Indeed, agrin immunoreaction was found to be highly selective to tumorous areas (Figure 4a). As this was a consistent observation, we propose that agrin immunohistochemistry might facilitate differentiation between regenerative and malignant nodules. In this regard, agrin may even be a better marker of malignant transformation than perlecan, because the latter

Figure 3 Agrin is localized in vascular and biliary basement membranes. Double immunofluorescence images; red: agrin (R\&D Systems), green: as indicated in the picture labels, blue: nuclei. (a, c) Human HCC; (b, d) DPC-treated rat liver. (a, b) Using endothelial markers, blood vessels in the fibrous septa (thick arrows) and tumor microvessels (thin arrows) appear as double positive structures. Agrin $+/$ endothelial- structures are bile ducts and ductuli (arrowheads). (c, d) As a complementary reaction, agrin is here paired with pancytokeratin. Here, double positive structures are bile ducts and ductuli (arrowheads); agrin + /CK - structures are blood vessels (thick arrows). Faintly agrin + tumor microvessels (thin arrow) can be observed inside the CK + area of a HCC nodule in (d). Scale bars, $50 \mu$ m. 

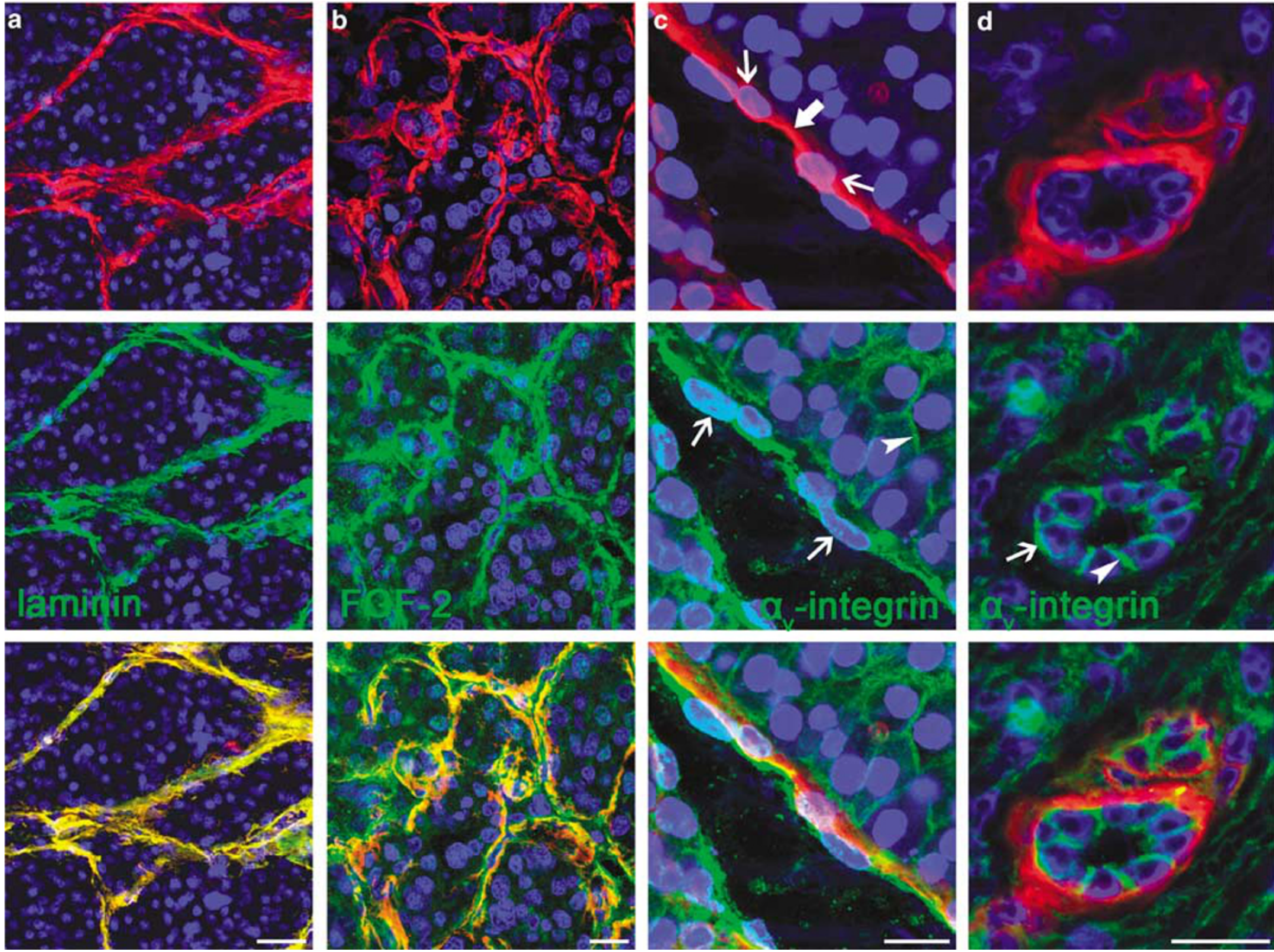

Figure 5 Putative molecular partners of agrin in the liver. (a-d) Double immunofluorescence on cryosections from human HCC (a-c) and cirrhotic liver (d); confocal images. Red: agrin (R\&D Systems), green: as specified in the picture labels, blue: nuclei. In the composed images, shown in the lower row, yellow color indicates colocalization or overlap. Agrin is thought to be immobilized in the basement membranes by binding to laminin (a); agrin, in turn, is capable of binding growth factors like FGF-2 (b). Here, agrin is shown to colocalize with the above two species in the microvascular basement membranes of the HCC. (c) $\alpha_{\mathrm{v}}$-Integrins, expressed by endothelial cells in a tumor blood vessel (thin arrows in the middle row), may serve as receptors for agrin deposited in the underlying basement membrane (thick arrow in the upper row). Agrin in this localization is probably secreted by pericytes (thin arrows in the upper row). Cancer cells, although to a lesser extent, also express $\alpha_{\mathrm{v}}$-integrin (arrowhead in the middle row). (d) Biliary epithelial cells express $\alpha_{\mathrm{v}}$-integrins on the whole length of their basolateral membrane domain (basal surface: thin arrow; lateral surface: arrowhead). The basal surface is lying on an agrin-containing basement membrane. Scale bars, $50 \mu \mathrm{m}$ (a), $25 \mu \mathrm{m}$ (b), $20 \mu \mathrm{m}$ (c, d).

could be occasionally observed in benign regenerative parenchymal nodules as well (Figure $4 \mathrm{~b}$ and c).

\section{Putative Molecular Partners of Agrin in the Liver}

Like in other organs, agrin in liver basement membranes is likely to be immobilized by binding to laminin (Figure 5a). Agrin, in turn, may immobilize and present growth factors: the heparan sulfate ligand basic fibroblast growth factor was seen to be concentrated in all agrin-positive basement membranes (Figure 5b). However, the relative importance of agrin compared with other basement membraneHSPGs in growth factor binding still needs to be determined.

Not only the growth factors bound to it, but agrin itself can also serve as a ligand for cell surface receptors expressed by cells that attach to agrin-containing basement membranes. $\alpha_{\mathrm{v}}$-Integrins,

Figure 6 Candidate cellular sources of agrin in the diseased liver. (a, d) Double immunofluorescence on cryosections from a human cirrhotic liver (a), a DPC-treated rat liver (b) and a human HCC (c, d); confocal images. Red: agrin (R\&D Systems), green: as indicated in the picture labels, blue: nuclei. Agrin and SMA immunoreactions overlap in the walls of blood vessels (thick arrows, a, b), allowing us to conclude that agrin in blood vessel walls is predominantly produced by vascular smooth muscle cells. As no SMA + cells surround bile ducts, agrin in biliary basement membranes is thought to be secreted by biliary epithelial cells. In the stroma of the HCC, both SMApositive mesenchymal cells and CD31-positive endothelial cells are seen adjacent to the basement membranes (c-d). Scale bars, $50 \mu \mathrm{m}$ (A), $25 \mu \mathrm{m}$ (B), $10 \mu \mathrm{m}(\mathbf{c}, \mathbf{d})$. 

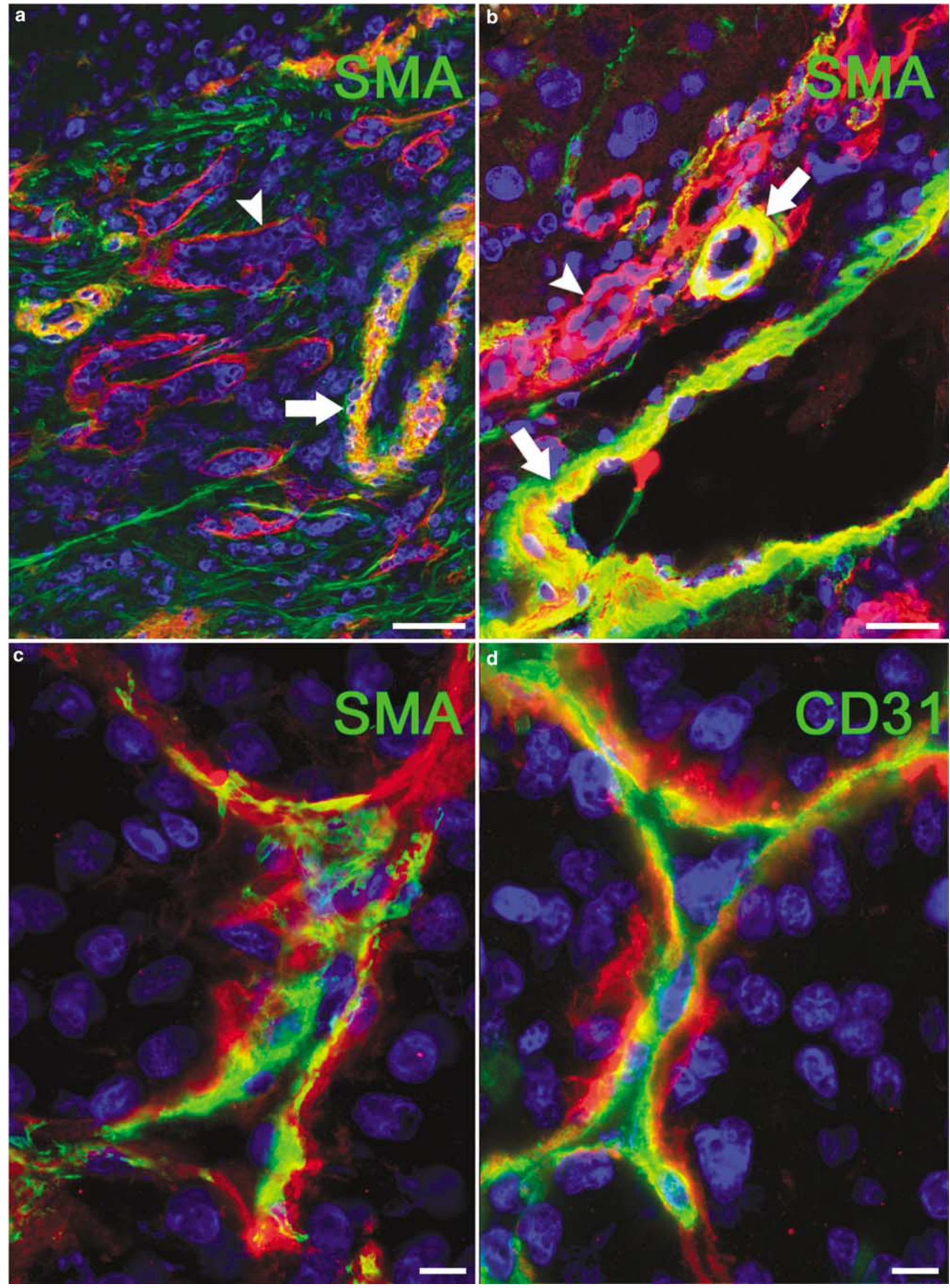
previously proven to interact with agrin, ${ }^{13,14}$ were found to be expressed by both endothelial cells and biliary epithelial cells (Figure 5c and d). The latter cell type exhibits $\alpha_{\mathrm{v}}$-integrin immunopositivity on the whole length of its basolateral surface (Figure $5 \mathrm{~d}$ ), which suggests a role for $\alpha_{\mathrm{v}}$-integrin independent from the attachment to the basement membrane. Also, $\alpha_{\mathrm{v}}$-integrin is expressed by cancer cells surrounded by a matrix that apparently lacks agrin (Figure 5c). Nevertheless, agrin and $\alpha_{\mathrm{v}}$-integrins are likely to interact on the basement membrane-cell interfaces in bile ducts and blood vessels.
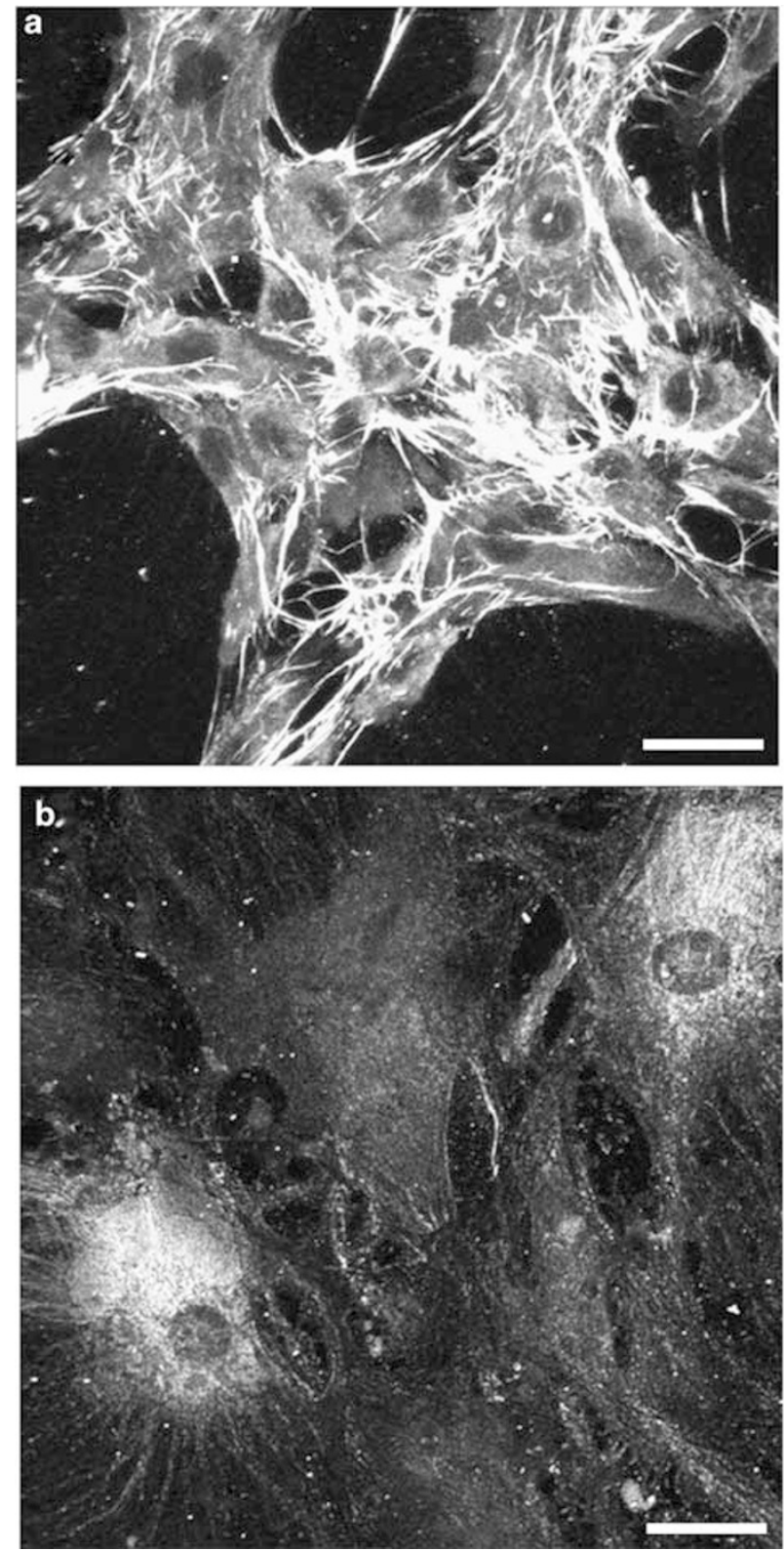

Figure 7 Agrin immunostaining of cultured rMFs and endothelial cells from human dermal microvasculature. By fluorescent immunocytochemistry (antibody: polyclonal, Santa Cruz R-20), myofibroblasts (a) appear to synthesize and secrete agrin more vigorously than endothelial cells (b). Scale bars, $20 \mu \mathrm{m}$.

\section{Candidate Cellular Sources of Agrin in the Diseased Liver}

In the walls of arteries and veins, the thickness of agrin immunoreaction, by simple visual estimation, correlates with the thickness of the musculature (eg see Figure $1 \mathrm{~b}$ ), and vascular smooth muscle cells perfectly overlap with agrin deposition (Figure 6a and b). Therefore, vascular smooth muscle cells are thought to secrete agrin into their enveloping basement membranes, and perhaps also into the endothelial basement membrane. On the other hand, bile ductules are not closely surrounded by smooth muscle $\alpha$-actin (SMA)-positive cells; still, basement membranes underlying biliary epithelia always showed a strong agrin immunostaining. Thus, biliary epithelial cells are additional independent candidates for agrin production.

Agrin in tumor microvessel basement membranes may originate from SMA-positive stromal myofibroblasts, from endothelial cells, or both (Figure 6c and d). By immunocytochemistry, rMFs seem to secrete agrin much more vigorously than microvascular endothelial cells do; however, the role of endothelial cells in agrin deposition cannot be excluded (Figure 7a and b). Isolated rMFs and HSCs were also tested for agrin expression by RT-PCR. Both cell types proved to synthesize agrin mRNA at high levels (Figure 8a). Moreover, agrin could be detected by immunoblotting in the supernatants of both rMF and HSC cultures (Figure 8b).
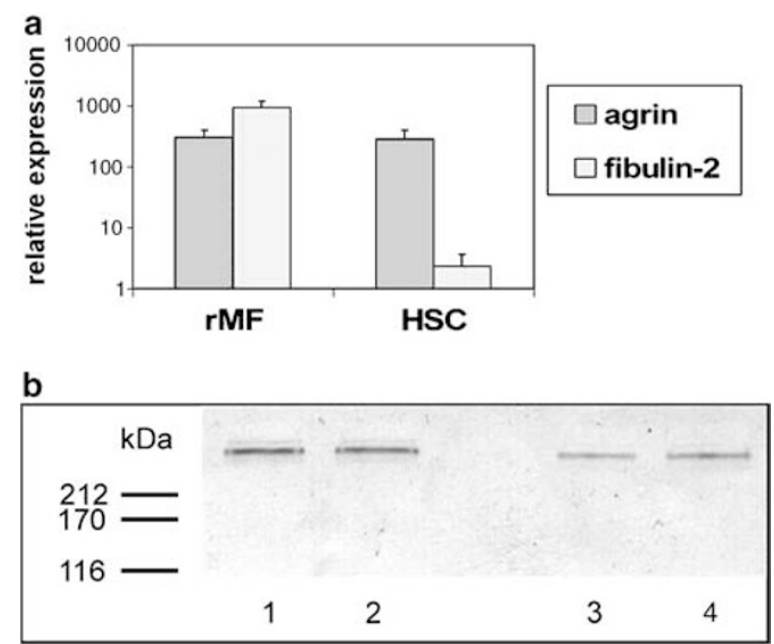

Figure 8 Expression of agrin by rat liver mesenchymal cells. (a) By quantitative real-time RT-PCR, both fibulin-2-positive myofibroblasts (rMF) and fibulin-2-negative HSC isolated from rat liver express agrin at high levels (relative to total RNA from normal rat liver, calibrated with ubiquitin C). (b) Agrin secreted into the culture medium by rMF (lanes 1-2) and HSC (lanes 3-4) can be detected by immunoblotting using the polyclonal antibody R-20 from Santa Cruz. 


\section{Discussion}

In the present study, agrin is described for the first time as a basement membrane component of blood vessel walls and bile ducts in the liver. We found, although in minor quantities, agrin in the healthy liver as well. Normally, agrin-containing structures are restricted to the relatively small portal areas. In cirrhosis, however, fibrogenesis is usually accompanied by ductular reaction and vascularization of the extending septa. The proliferation of bile ductules and blood vessels, both having basement membranes rich in agrin, leads to the accumulation of this PG initially present only in marginal amounts in the liver.

Agrin was found to be a point of difference between the basement membranes of HCC microvasculature and the sinusoidal walls in the regenerative nodules. Agrin appears in the basement membrane of tumor microvasculature, while sinusoidal walls, as a rule, lack this PG. Perlecan, a cognate basement membrane-HSPG exhibits no such a strict selectivity. The expression of agrin in the HCC microvasculature may be induced by tumor-specific, tumor cell-derived mediators: the tumor-induced stromal expression of HSPGs is a well-known phenomenon. Syndecan-1 appears in the stroma of several carcinoma types, ${ }^{28-32}$ and syndecan-3 was identified in HCC stromal vessels. ${ }^{33}$ These HSPGs, along with perlecan, are believed to be involved in tumoral angiogenesis and in stromal cell proliferation.

Agrin in the liver seems to be of heterogeneous origin. Agrin in the wall of blood vessels and probably in the basement membrane of tumor microvasculature is produced by SMA-positive cells, which are vascular smooth muscle cells in macroscopic arteries and veins and myofibroblastlike cells acting as pericytes in microvessels. Agrin expression at the mRNA level has been detected in smooth muscle cells, ${ }^{34}$ and in our experiments, myofibroblasts and HSCs isolated from rat liver were also found to synthesize agrin. As a potential source of agrin in the endothelial basement membrane, endothelial cells should also be taken into account, despite the weak in vitro agrin positivity of human dermal microvascular endothelial cells. Smaller bile ducts, unlike blood vessels, are not surrounded by SMA-positive cells. Bile duct basement membranes, however, always contain agrin, which here is presumably produced by the biliary epithelium in an autonomous manner.

Whether in vascular or bile duct basement membranes, agrin in the liver was consistently found in close spatial proximity with $\alpha_{\mathrm{v}}$-integrins. An important role in angiogenesis is attributed to this species, ${ }^{35,36}$ while its contribution to normal bile duct development and pathologic ductular reaction has not been described. It is also indicative for a possible proangiogenic effect of agrin that, beside perlecan, agrin is also able to bind various growth factors and present them to their receptors; basic fibroblast growth factor, a potent stimulator of angiogenesis was found in agrin-containing basement membranes.

Taken together, these findings suggest that agrin might play a role in vascular and ductular proliferation characteristic of cirrhosis, and may promote tumor progression by supporting stromal cell growth and neoangiogenesis in the HCC. Remarkably, agrin is selective to the tumor microvessel walls when compared to the sinusoidal walls of cirrhotic regenerative nodules; thus, agrin immunohistochemistry may facilitate detection of malignant nodules. Finally, since the accumulation of agrin can be accurately and reliably reproduced in a rodent model of liver cirrhosis and HCC, the way is paved for testing the above hypotheses in vivo in the future.

\section{Acknowledgements}

We express our gratitude to Peter Bannasch at Deutsches Krebsforschungszentrum, Heidelberg, Germany for his contribution to the pathological liver specimens, and to Krisztina Egedi, Júlia O Nagy, Zsuzsanna Madarassy and Sándor Paku for their invaluable help. Special thanks to Tamás Janáky and his group at the Department of Medical Chemistry, Faculty of Medicine, University of Szeged for the mass spectrometry analysis. This work was supported by National Research and Development Program, Hungary (NKFP) NKFP-1A/ 0023/2002; Hungarian Scientific Research Fund (OTKA) T 042672; Medical Research Council, Hungary (ETT) 255/2003; Deutsche Forschungsgemeinschaft (DFG) SFB402TPC6 and GRK335.

\section{References}

1 Gallai M, Kovalszky I, Knittel T, et al. Expression of extracellular matrix proteoglycans perlecan and decorin in carbon-tetrachloride-injured rat liver and in isolated liver cells. Am J Pathol 1996;148:1463-1471.

2 Kovalszky I, Nagy P, Szende B, et al. Experimental and human liver fibrogenesis. Scand J Gastroenterol Suppl 1998;228:51-55.

3 Dudas J, Kovalszky I, Gallai M, et al. Expression of decorin, transforming growth factor-beta 1, tissue inhibitor metalloproteinase 1 and 2, and type IV collagenases in chronic hepatitis. Am J Clin Pathol 2001;115:725-735.

4 Hogemann B, Edel G, Schwarz K, et al. Expression of biglycan, decorin and proteoglycan-100/CSF-1 in normal and fibrotic human liver. Pathol Res Pract 1997;193:747-751.

5 Kovalszky I, Schaff Z, Jeney A. Potential markers (enzymes, proteoglycans) for human liver tumors. Acta Biomed Ateneo Parmense 1993;64:157-163.

6 Rescan PY, Loreal O, Hassell JR, et al. Distribution and origin of the basement membrane component perlecan 
in rat liver and primary hepatocyte culture. Am J Pathol 1993;142:199-208.

7 Aviezer D, Hecht D, Safran M, et al. Perlecan, basal lamina proteoglycan, promotes basic fibroblast growth factor-receptor binding, mitogenesis, and angiogenesis. Cell 1994;79:1005-1013.

8 Jiang X, Couchman JR. Perlecan and tumor angiogenesis. J Histochem Cytochem 2003;51:1393-1410.

9 Iozzo RV. The biology of the small leucine-rich proteoglycans. Functional network of interactive proteins. J Biol Chem 1999;274:18843-18846.

10 Bezakova G, Ruegg MA. New insights into the roles of agrin. Nature Rev Mol Cell Biol 2003;4:295-309.

11 Groffen AJ, Veerkamp JH, Monnens LA, et al. Recent insights into the structure and functions of heparan sulfate proteoglycans in the human glomerular basement membrane. Nephrol Dial Transplant 1999;14: 2119-2129.

12 Gesemann M, Brancaccio A, Schumacher B, et al. Agrin is a high-affinity binding protein of dystroglycan in non-muscle tissue. J Biol Chem 1998;273:600-605.

13 Martin PT, Sanes JR. Integrins mediate adhesion to agrin and modulate agrin signaling. Development 1997;124:3909-3917.

14 Burgess RW, Dickman DK, Nunez L, et al. Mapping sites responsible for interactions of agrin with neurons. J Neurochem 2002;83:271-284.

15 McMahan UJ. The agrin hypothesis. Cold Spring Harb Symp Quant Biol 1990;55:407-418.

16 Cole GJ, Halfter W. Agrin: an extracellular matrix heparan sulfate proteoglycan involved in cell interactions and synaptogenesis. Perspect Dev Neurobiol 1996;3:359-371.

17 Kroger S, Schroder JE. Agrin in the developing CNS: new roles for a synapse organizer. News Physiol Sci 2002;17:207-212.

18 Barber AJ, Lieth E. Agrin accumulates in the brain microvascular basal lamina during development of the blood-brain barrier. Dev Dyn 1997;208:62-74.

19 Khan AA, Bose C, Yam LS, et al. Physiological regulation of the immunological synapse by agrin. Science 2001;292:1681-1686.

20 Groffen AJ, Ruegg MA, Dijkman H, et al. Agrin is a major heparan sulfate proteoglycan in the human glomerular basement membrane. J Histochem Cytochem 1998;46:19-27.

21 Knittel T, Kobold D, Saile B, et al. Rat liver myofibroblasts and hepatic stellate cells: different cell populations of the fibroblast lineage with fibrogenic potential. Gastroenterology 1999;117:1205-1221.

22 Desmouliere A, Guyot C, Gabbiani G. The stroma reaction myofibroblast: a key player in the control of tumor cell behavior. Int J Dev Biol 2004;48:509-517.

23 Zalatnai A, Lapis K. Simultaneous induction of liver cirrhosis and hepatocellular carcinomas in F-344 rats: establishment of a short hepatocarcinogenesis model. Exp Toxicol Pathol 1994;46:215-222.

24 Neubauer K, Knittel T, Aurisch S, et al. Glial fibrillary acidic protein-a cell type specific marker for Ito cells in vivo and in vitro. J Hepatol 1996;24:719-730.

25 Saile B, Matthes N, Neubauer K, et al. Rat liver myofibroblasts and hepatic stellate cells differ in CD95-mediated apoptosis and response to TNF-alpha. Am J Physiol Gastrointest Liver Physiol 2002;283: G435-G444.

26 Lyon M, Gallagher JT. Purification and partial characterisation of the major cell-associated heparan sulphate proteoglycan of rat liver. Biochem J 1991; 273:415-422.

27 Kemeny E, Fillit HM, Damle S, et al. Monoclonal antibodies to heparan sulfate proteoglycan: development and application to the study of normal tissue and pathologic human kidney biopsies. Connect Tissue Res 1988;18:9-25.

28 Stanley MJ, Stanley MW, Sanderson RD, et al. Syndecan-1 expression is induced in the stroma of infiltrating breast carcinoma. Am J Clin Pathol 1999;112:377-383.

29 Bayer-Garner IB, Dilday B, Sanderson RD, et al. Syndecan-1 expression is decreased with increasing aggressiveness of basal cell carcinoma. Am J Dermatopathol 2000;22:119-122.

30 Wiksten JP, Lundin J, Nordling S, et al. Epithelial and stromal syndecan-1 expression as predictor of outcome in patients with gastric cancer. Int J Cancer 2001; 95:1-6.

31 Ito $\mathrm{Y}$, Yoshida H, Nakano K, et al. Syndecan-1 expression in thyroid carcinoma: stromal expression followed by epithelial expression is significantly correlated with dedifferentiation. Histopathology 2003;43:157-164.

32 Mukunyadzi P, Liu K, Hanna EY, et al. Induced expression of syndecan-1 in the stroma of head and neck squamous cell carcinoma. Mod Pathol 2003; 16:796-801.

33 Roskams T, De Vos R, David G, et al. Heparan sulphate proteoglycan expression in human primary liver tumours. J Pathol 1998;185:290-297.

34 Tsen G, Napier A, Halfter W, et al. Identification of a novel alternatively spliced agrin mRNA that is preferentially expressed in non-neuronal cells. J Biol Chem 1995;270:15934-15937.

35 Eliceiri BP, Cheresh DA. The role of alphav integrins during angiogenesis: insights into potential mechanisms of action and clinical development. J Clin Invest 1999;103:1227-1230.

36 Trikha M, Zhou Z, Nemeth JA, et al. CNTO 95, a fully human monoclonal antibody that inhibits alphav integrins, has antitumor and antiangiogenic activity in vivo. Int J Cancer 2004;110:326-335. 